\title{
Bearing Fault Detection Using DWT and CNN
}

doi : https://doi.org/10.32628/CSEIT2174116

\section{Shubham D. Paranjape*, Prof. J. A. Gaikwad}

Department of Instrumentation Engineering, Vishwakarma institute of technology, Pune, Maharashtra, India

Article Info

Volume 7, Issue 4

Page Number: 476-483

Publication Issue :

July-August-2021

\section{Article History}

Accepted : 01 Aug 2021

Published : 08 Aug 2021

\section{ABSTRACT}

Bearing is a key component of satellite inertia actuators such as moment wheel assemblies (MWAs) and control moment gyros (CMGs), and its operating state is directly related to the performance and service life of satellites. However, because of the complexity of the vibration frequency components of satellite bearing assemblies and the small loading, normal running bearings normally present similar fault characteristics in long-term ground life experiments, which makes it difficult to judge the bearing fault status. There are various methods introduced for condition monitoring such as vibration analysis, temperature analysis, wear and debris analysis, image processing etc. Among this image analysis is found to be the most effective method for detection of machine faults. This paper proposes an automatic fault diagnosis method for bearings based on a DWT and CNN.

Keywords : DWT, CNN classifier, image processing, Bearing, MWA

\section{INTRODUCTION}

Bearing is a rotating machine element, used to enable rotational or linear movement, while reducing friction \& handling stress. Rolling element bearing carries a load by placing rolling elements such as balls or rollers between two bearing rings called races. Bearings are used in household applications to heavy duty industrial equipment due to their simple construction and high reliability. Rotating machines have to undergo regular maintenance like break down, preventive and predictive maintenance to avoid fault occurrence. Faults in rotating machines are due to faults developed in stator, rotor, bearing. $40 \%$ faults are due to bearings $38 \%$ are due to stator, $10 \%$ faults are due to rotor. The main source of system failure is bearing. The performance of the machine depends upon health of bearing. Therefore, Condition monitoring $(\mathrm{CM})$ is necessary. The reasons behind increased vibrations are misalignment, looseness, unbalance, bend shaft, worn gears or bearing. The result of these vibration causes is pitting, flaking, smearing and fracture. To identify the reason of vibration, long term experience is needed which everyone does not have it. Since need of modern instrumentation for measuring vibration on rotating machine is generated to minimize need of good, longterm experience as well as to detect developing problems which are out of scope of human perception. As human perception vary person to person so it becomes crucial that there is a requirement of standard method for early fault detection and identification from vibration signal. So, the studies for early detection, diagnosis and classification of faults in 
engineering studies become inevitable. Condition monitoring is Process of monitoring a parameter of condition in order to identify significant change which is indicative of a developing fault. CM of electrical motors can increase their life, productivity, and safety. Condition monitoring techniques are Vibration analysis, Wear Debris Analysis, Oil condition Analysis, Acoustic Emission, \& Thermography. As compare to other techniques, Vibration analysis method is very easy to implement. Vibration analysis carries rich information on machine health conditions \& with this method early detection of faults is possible also vibration analysis decreases down time of machine, cost effective and can apply for non-stationary signals.

Preventive maintenance is a growing trend in industrial operations. One critical area of preventive maintenance includes vibration monitoring. Typically, the most critical area for analysis is directed at the bearings. Hence it is important to go for vibration analysis of bearing faults. $40 \%$ issues in rotating machines are due to faults developed in bearing. Bearing is a main source of system failure. The performance of the machine depends upon health of bearing. Bearing is cheap, but the failure of bearing is costly. Image analysis not only minimizes the need for extensive experience, but makes it possible to detect developing problems and reduces maintenance cost.

\section{LITERATURE SURVEY}

Condition monitoring involves the continuous or periodic assessment of the condition of a plant or a machinery component while it is running. Basically, condition monitoring is the process of monitoring some parameters from the machinery, such that a significant change in the parameter can give information about the health of the machinery. Condition Monitoring is most frequently used as a Predictive or Condition Based Maintenance technique.
There are various techniques of predictive maintenance that can also be used, including the use of the Human Senses (look, listen, feel, smell etc.) but it varies person to person. So, there is need of such method that is uniform everywhere which allows maintenance to be scheduled other actions to be taken to avoid the consequences of failure before failure occurs. It allows an organization to schedule production shut down for repairs, inspection and maintenance.

There are few CM techniques explained below which are usually used to check health of machines Vibration Analysis: Every healthy bearing produces certain number of vibrations and abnormal vibration in rotating machinery is the first indication of rotating component failure. Amplitude of vibration signal indicates severity of fault and frequency indicates source of fault/ defect. Vibration Analysis is used to detect early precursors to machine failure, allowing machinery to be repaired or replaced before an expensive failure occurs. It can be employed to diagnose all kinds of faults which may be localized or distributed. Vibration analysis does not require tearing a machine to find out its condition. When a machine condition fault comes up, a repair is scheduled when its needed not before and not too late. Wear Debris Analysis: Wear generates debris. The debris comes in a wide variety of sizes and shapes. Wear debris represents loss of geometric accuracy of moving contacting parts. Wear debris analysis is carried out on used greases by extracting magnetic particles from the sample using a magnet. Microscopic analysis of the sample identified numerous small and large spherical particles. Research has shown that spherical wear debris can reveal the severity of rolling contact fatigue wear. Because large spherical particles (50 microns) are the product of high metal to metal contact and high frictional temperature, their presence is considered a supporting symptom for assessing the wear severity levels. Wear particles were considered to be a critical alarm indicating the need to change the bearing before a forced outage occurs. 
Oil Condition Analysis: Oil Analysis is the analytical results of a lubricants property, suspended contaminants and wears debris. Oil Analysis is performed during routine preventative maintenance to provide meaningful and accurate information on lubricants and the machine condition. It is the best tool to reveal vital information about equipment condition and performance. It provides information for two important aspects; the condition of the oil being analysed in laboratory and report is generated.

Acoustic Emission: Acoustic emission is the phenomenon of radiation of acoustic (ultrasound) waves in solids that occurs when a material undergoes irreversible changes in its internal structure. With acoustics condition monitoring, a machine running in a good condition has a stable noise spectrum. Spectrum changes when the condition changes. Identification of noise source and comparing its spectrum with that of a stable spectrum of a machine or plant in good condition. The detection of cracks is the prime application of acoustic emission. This is used as tool for condition monitoring of shaft cracks and bearing faults.

Thermography: Thermography is a diagnostic procedure used to detect temperature variations in machinery that are cause for concern. Pictures of machinery are taken using a thermographic camera. Areas of the equipment that are over heating can be spotted immediately with one picture, which would otherwise never have seen with the naked eye. A report is received with the pictures and recommendations, allowing correcting the problem before it can cause any damage.

Image processing is a method to perform some operations on an image, in order to get an enhanced image or to extract some useful information from it. It is a type of signal processing in which input is an image and output may be image or characteristics/features associated with that image. Nowadays, image processing is among rapidly growing technologies. It forms core research area within engineering and computer science disciplines too. Image Processing is a technique to improve raw images received from cameras or sensors placed on satellites, space probes and aircrafts or pictures taken in normal life for various applications. Various techniques have been developed in Image Processing during the last five decades. Most of the techniques are developed for enhancing images obtained from unmanned spacecrafts, space probes and military inspection flights. Image Processing systems are becoming popular due to easy availability of powerful personnel computers, large size memory devices, graphics software. Image Processing is used in various applications such as Remote Sensing, Medical Imaging, Textiles, Material Science, Military, Film industry, Document processing. Graphic arts the common steps in image processing are image scanning, storing, enhancing and interpretation.

\section{PROPOSED SYSTEM}

\section{A. Algorithm \\ 1) DWT}

The Wavelet Transform is a powerful tool to analyse power system transients. Similar to a Fourier

Transform (FT), wavelet transform can decompose the signal into different frequencies, and more than FT, by using wavelet transform, the signal can be broken up into shifted and scaled versions of the mother wavelet. There are two forms of wavelet transform; the continuous wavelet (CWT) and discrete wavelet transform (DWT). The Continuous Wavelet Transform (CWT) of a signal $\mathrm{x}(\mathrm{t})$ is defined as:

$$
\operatorname{CWT}_{\psi} x(a, b)=\frac{1}{\sqrt{|a|}} \int_{-\infty}^{+\infty} x(t) \Psi^{*}\left(\frac{t-b}{a}\right) d t
$$

where $\Psi(t)$ is called the mother wavelet, the scaling parameters $\mathrm{a}$ and $\mathrm{b}$ determines the oscillatory frequency, the length of the wavelet and the shifting position respectively. The application of wavelet transform in engineering areas usually requires the 
discrete wavelet transform, the equation of the discrete wavelet transform is given by:

$$
\operatorname{DWT}(k, n, m)=\frac{1}{\sqrt{a_{0}^{m}}} \sum x[n] \Psi\left(\frac{k-n b_{0} a_{0}^{m}}{a_{0}^{m}}\right)
$$

In DWT, the mother wavelet becomes $\Psi \mathrm{m}, \mathrm{n}(\mathrm{t})=\mathrm{a}$ $-\mathrm{m} / 2 \quad 0 \quad \Psi(\mathrm{a}-\mathrm{m} 0 \mathrm{t}-\mathrm{nb} 0)$, where $\mathrm{m}$ indicates frequency localization and $\mathrm{n}$ denotes time localization. 2) $\mathrm{CNN}$

Over the last decade, tremendous progress has been made in the field of artificial neural networks. Deep layered convolutional neural networks (CNN) have demonstrated state of the art results on many machine learning problems, especially image recognition tasks. CNN is one of artificial neural networks which have distinctive architectures as shown in Figure. 1; Input data of CNN are usually RGB images (3 channels) or gray scale images (1 channel). Several convolutional or pooling layers (with or without activation functions) follows the input layer. For classification problems, one or more full connection (FC) layers are often employed. The final layer outputs prediction values (such as posterior probability or likelihood) for kinds of objects where the input image should be classified in.

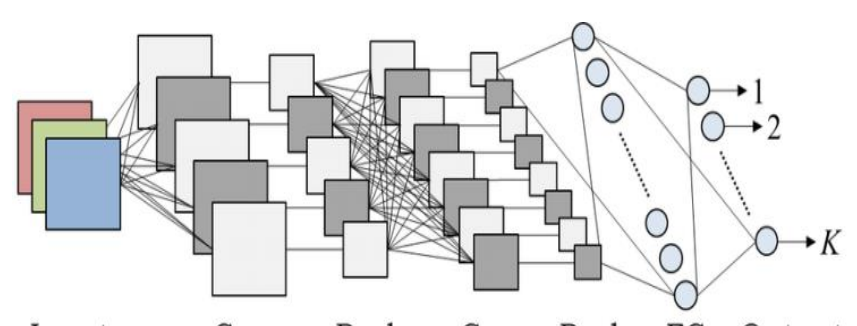

Input Conv Pool Conv Pool FC Output

Figure 1. CNN architecture.

Each layer of CNN can have a certain activation function which controls amount of output value to propagate its next layer. For intermediate layers, the rectified linear unit (ReLU)

$$
f\left(a_{i}^{l}\right)=\max \left(0, a_{i}^{l}\right)
$$

Note that all $i \in R$ is a sum of signals received by the $i$ th unit in the 1-th intermediate layer. Meanwhile, for the last layer, the soft-max functions often used to obtain probabilistic outputs.

$$
f_{k}(\boldsymbol{z})=\frac{\exp \left(z_{k}\right)}{\sum_{\kappa=1}^{K} \exp \left(z_{\kappa}\right)},
$$

Note that $\mathrm{z}$ is a $\mathrm{K}$-dimensional vector where $\mathrm{zk}$ is a sum of signals received by the $\mathrm{k}$-th unit in the last layer. Since the function is non-negative and has the unit sum property $(\mathrm{kfk}(\mathrm{z})=1)$, the value of $\mathrm{fk}$ implies a class posterior probability that an input data belongs to the $\mathrm{k}$-th class. Therefore, by using the soft max function in the output layer, CNN can act a role of probability estimators for the object classification problems. As one of the distinctive properties of CNN, they have consecutive multiple feature representations which are automatically organized in each convolutional layer through the training using given labeled instances. In spite of this interesting situation, typical dimensionality reduction methods (such as PCA) will visualize each feature representation individually, without regarding the relationships between those consecutive features.

\section{CNN Architecture}

- Convolutional Layer: It applies 14 5x5 filters (extracting 5x5-pixel sub-regions),

- $\quad$ Pooling Layer: This will perform max pooling with a $2 \times 2$ filter and stride of 2 (which specifies that pooled regions do not overlap).

- Convolutional Layer: It applies 36 5x5 filters, with ReLU activation function

- $\quad$ Pooling Layer: Again, performs max Pooling with a $2 \times 2$ filter and stride of 2 .

- $\quad 1,764$ neurons, with the dropout regularization rate of 0.4 (where the probability of 0.4 that any given element will be dropped in training) 
- Dense Layer (Logits Layer): There are ten neurons, one for each digit target class (0-9).

Important modules to use in creating a $\mathrm{CNN}$ :

- Conv2d (). Construct a two dimensional convolutional layer with the number of filters, filter kernel size, padding, and activation function like arguments.

- max_pooling2d (). Construct a two dimensional pooling layer using the max pooling algorithm.

- Dense (). Construct a dense layer with the hidden layers and units.

\section{B. ARCHITECTURE}

Image from database is taken as input image. The aim of preprocessing is an improvement of the image data that suppresses unwanted distortions (i.e. noise removing) or enhances some image features important for further processing. It is sometimes of interest to process a single subregion of an image, leaving other regions unchanged. This is commonly referred to as region-of-interest (ROI) processing.

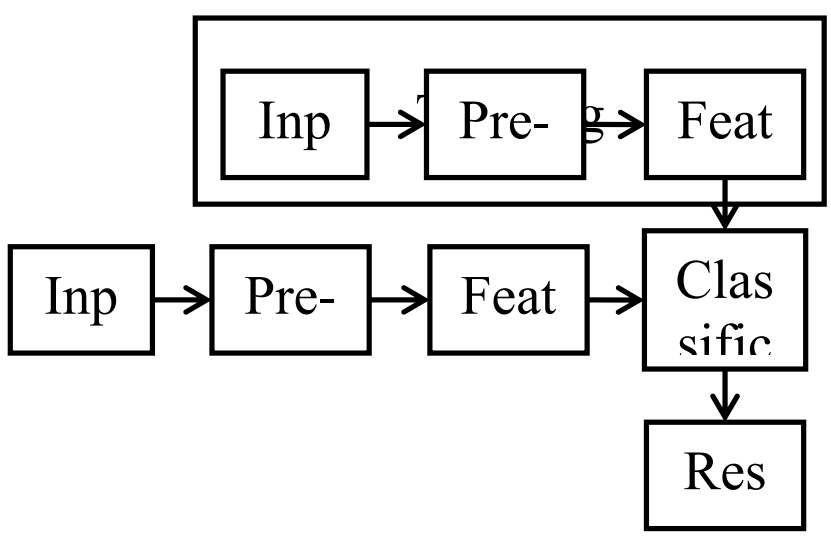

Figure 2. Block diagram of proposed system

A region of interest (ROI) is a portion of an image that you want to filter or perform some other operation on. ROI is extracted from input image in preprocessing step. Feature extraction: features like mean, kurtosis, skewness is extracted from preprocessed image.
Following operations are performed to extract features from input image

a) Contrast

$$
\text { contrast }=\sum_{i, j=0}^{N-1} P_{i j}(i-j)^{2}
$$

\section{b) Correlation}

Correlation is a method for establishing the degree of probability that a linear relationship exists between two measured quantities. Pearson's correlation coefficient, $r$, was the first formal correlation measure and is widely used in statistical analysis, pattern recognition and image processing. For monochrome digital images, the Pearson's correlation coefficient is defined as

$$
r=\frac{\sum_{i}\left(x_{i}-x_{m}\right)\left(y_{i}-y_{m}\right)}{\sqrt{\sum_{i}\left(x_{i}-x_{m}\right)^{2}} \sqrt{\sum_{i}\left(y_{i}-y_{m}\right)^{2}}}
$$

c) Energy

$$
\text { energy }=\sum_{\mathrm{i}, \mathrm{j}=0}^{\mathrm{N}-1}\left(\mathrm{P}_{\mathrm{ij}}\right)^{2}
$$

d) Homogeneity

$$
\text { Homogenity }=\sum_{\mathrm{i}, \mathrm{j}=0}^{\mathrm{N}-1} \frac{P_{i j}}{1+(i-j)^{2}}
$$

e) Mean

In contest of image processing filtering using mean is classified as spatial filtering and used for noise reduction. In this section we have discussed about various type of mean and analysed their use for removing various type of noise in image processing.

$$
f(x, y)=\frac{1}{m n} \sum_{(r, c) \in W} g(r, c)
$$

Where ' $g$ ' is the noisy image, $f(x, y)$ is the restored image, and ' $r$ ' and ' $c$ ' are the row and column coordinates respectively, within a window ' $\mathrm{W}$ ' of size ' $m \times n$ ' where the operation takes place. 
f) Standard Deviation

It is a most widely used measure of variability or diversity used in statistics. In terms of image processing, it shows how much variation or "dispersion" exists from the average (mean, or expected value). A low standard deviation indicates that the data points tend to be very close to the mean, whereas high standard deviation indicates that the data points are spread out over a large range of values. Mathematically standard deviation is given by

$$
\begin{aligned}
& \text { standard deviation } \\
& =\sqrt{\frac{1}{m n-1} \sum_{(r, c) \in W}\left(g(r, c)-\frac{1}{m n-1} \sum_{(r, c) \in W} g(r, c)\right)^{2}}
\end{aligned}
$$

A standard deviation filter calculates the standard deviation and assigns this value to the center pixel in the output map. As it has capability in measuring the variability, it can be used in edge sharpening, as intensity level get changes at the edge of image by large value.

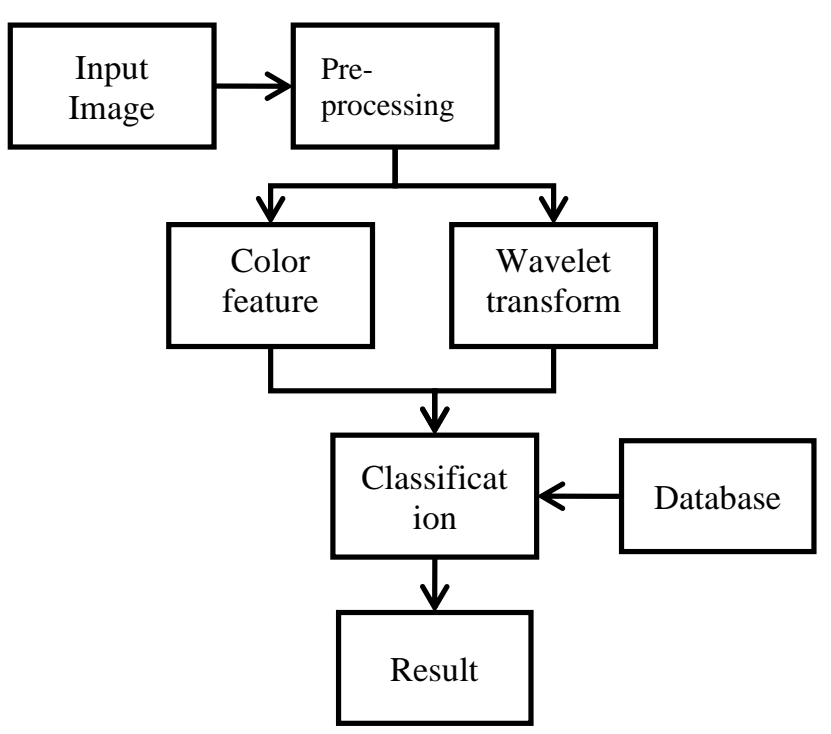

Figure 3. Flow chart of Proposed system

Here the Color related features are extracted and DWT (discrete wavelet transforms) is applied. Further
CNN algorithm is applied on extracted intensity to classify image of bearing and to detect faults in bearing.

\section{RESULT}

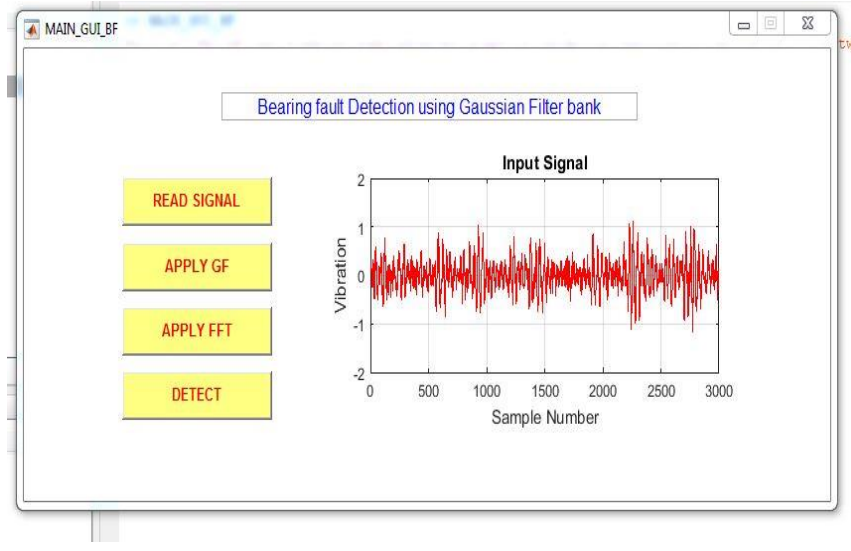

Figure 4. Read Signal

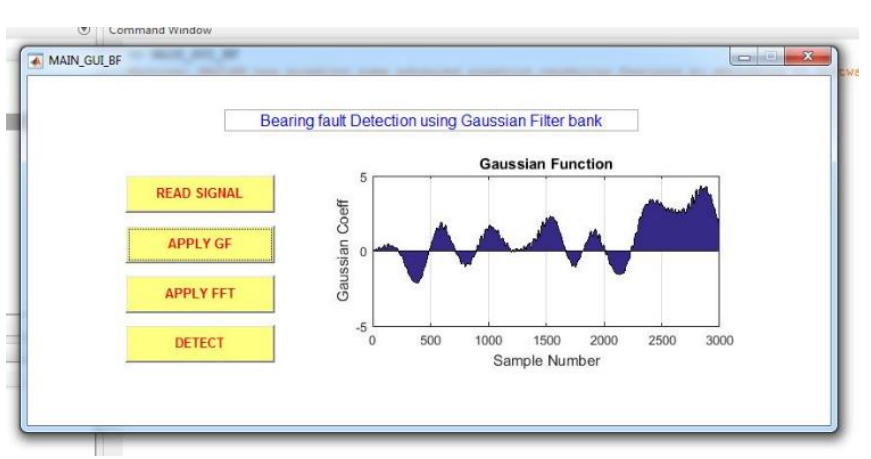

Figure 5. Apply GF

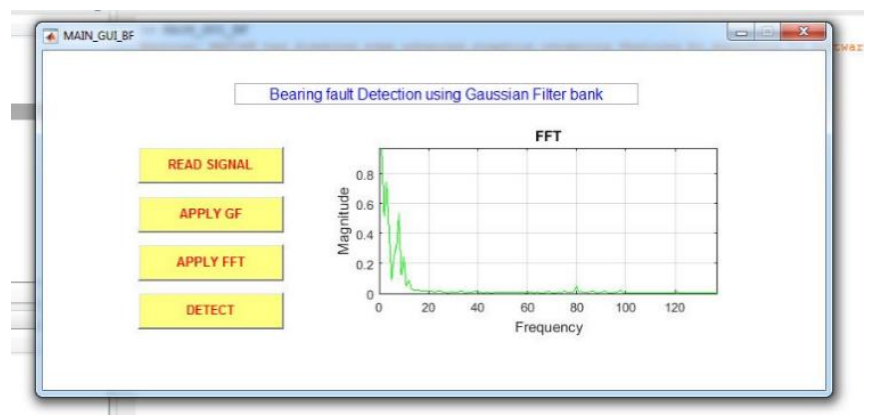

Figure 6. Apply FFT 


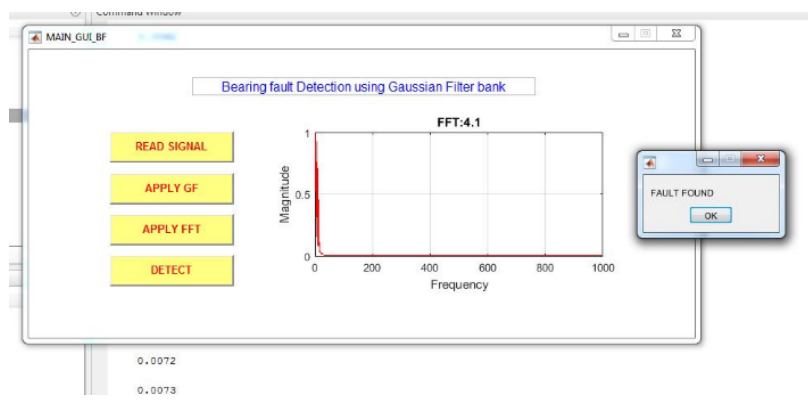

Figure 7. Detect

\section{CONCLUSION}

Analysis of image of bearing is one of the best condition monitoring techniques is used to detect and diagnose the existence of faults in rolling bearing of electric motor. Faults in SKF make bearing are artificially created and detected using machine learning technique such as $\mathrm{CNN}$. Compare to other condition monitoring techniques image analysis is quite effective technique to detect faults in rolling bearing in electric motor. Machine Learning is effective condition monitoring technique which decrease down time of machine, more cost effective and can apply for non-stationary signals.

\section{VI.REFERENCES}

[1] Pravesh Durkhure, Akhilesh Lodwal INTERNATIONAL JOURNAL OF ENGINEERING SCIENCES \& RESEARCH TECHNOLOGY Fault Diagnosis of Ball Bearing using Time Domain Analysis and Fast Fourier Transformation, Indore (2014)

[2] Lakshmi Pratyusha, ShanmukhaPriya, VPS Naidu, Bearing Health Condition Monitoring: Time Domain Analysis International Journal of Advanced Research in Electrical, Electronics and Instrumentation Engineering December (2014).

[3] Yogita K Chaudhari Vibration Analysis for Bearing Fault Detection in Electrical Motors, ICNSC-14, (2014).
[4] P. G. Kulkarni, A. D. Sahasrabudhe Application of Wavelet Transform for Fault Diagnosis of Rolling Element Bearings (2013).

[5] Milind Natu, Bearing Fault Analysis Using Frequency Analysis and Wavelet Analysis, International Journal of Innovation, Management and Technology, Vol. 4, No. 1, February (2013).

[6] BogdanBetea, LiviuTomesc, and Petru Dobra Automatic Control Department Technical University of Romania, Bearing Defects Diagnosis Based on Gaussian Filtering Shock Detection and Power Spectrum of Envelope (2013).

[7] Bogdanbetea, mircea-cristiangherman, monicaborda, petrudobraBearing defects signals demodulation using shock filters (2012).

[8] D. H. Pandya, S. H. Upadhyay, S. P. Harsha., ANN based fault diagnosis of rolling element bearing using time-frequency domain feature International Journal of Engg Science and Technology (IJEST) Vol. 4 No.06 June (2012).

[9] Xiang-jun Chen, Zhan-fengGao (2011) Data processing based on wavelet analysis in structure health monitoring system of Computers, vol. 6 no.12, pp. 2686-26

[10] J. Chebil, G. Noel et al (2009) Wavelet decomposition for the detection and diagnosis of faults in rolling element bearings Jordan-J of Mechanical and Industrial Engineering, vol.3, pp. 260-267 (1986).

[11] Hongyu Yang, Joseph Mathew and Lin Ma Vibration Feature Extraction Techniques for Fault Diagnosis of Rotating Machinery -A Literature Survey (2003).

[12] Tandon N and Choudhury A, (1999), A review of vibration and acoustic measurement methods for the detection of defects in rolling element bearings. Tribology International

[13] K. Chatfield, K. Simonyan, A. Vedaldi, and A. Zisserman. Return of the devil in the details: 
Shubham D. Paranjape et al Int. J. Sci. Res. Comput. Sci. Eng. Inf. Technol, July-August-2021, 7 (4) : 476-483

Delving deep into convolutional nets. arXiv preprint arXiv:1405.3531, 2014

\section{Cite this article as :}

Shubham D. Paranjape, Prof. J. A. Gaikwad, "Bearing Fault Detection Using DWT and CNN", International Journal of Scientific Research in Computer Science, Engineering and Information Technology (IJSRCSEIT), ISSN : 2456-3307, Volume 7 Issue 4, pp. 476-483, July-August 2021. Available at doi $\quad$ : https://doi.org/10.32628/CSEIT2174116 Journal URL : https://ijsrcseit.com/CSEIT2174116 